\title{
LA INVESTIGACIÓN DE FRAUDES EN LAS ORGANIZACIONES Y EL ROL DE AUDITORÍA INTERNA
}

\section{THE INVESTIGATION OF FRAUDS IN ORGANIZATIONS AND THE ROLE OF INTERNAL AUDIT}

\author{
Diego Marcelo Mantilla Garcés', Nancy Rocio Herrera Herrera²
}

\begin{abstract}
Resumen
La transparencia, la actuación frente al fraude y sobre todo de qué manera evitar la impunidad, situación muy grave para las organizaciones y el Estado, se han convertido tanto en los sectores públicos como privados en demandas sociales. Según la Encuesta sobre fraude y delito económico 2018, de PriceWaterhouseCoopers México (2018, p. 7) indica que del "porcentaje de organizaciones que experimentaron delitos económicos (2016-2018), el mayor incremento en delitos económicos se registró en América Latina, con un alza de 25 puntos porcentuales en comparación con 2016, paises de Europa del Este y Asia Pacífico experimentaron un aumento de 14 y 16 puntos porcentuales, respectivamente". Para esta investigación se utilizaron los métodos cualitativo (estudio de casos) y analítico con el objetivo de contribuir a la generación de protocolos de actuación frente al fraude mediante la presentación de casos acontecidos en compañías y una recopilación de estándares y publicaciones que define la importancia y el rol de la auditoría interna en la investigación de fraudes.
\end{abstract}

\section{Palabras clave}

Transparencia, fraude, impunidad, protocolo.

Clasificación JEL: H26, L21, M42

\begin{abstract}
Transparency, action against fraud and above all avoid a very serious issue for organizations and the state as strong as fraud itself is impunity, has become both the public and private sectors in a social demand. According to the Survey on fraud and economic crime 2018, Mexico edition of PriceWaterhouseCoopers (2018) indicates that of the percentage of organizations that experienced economic crimes (2016-2018) the greatest increase in economic crimes was registered in Latin America, with a 25 percentage point increase in comparison with 2016, countries in Eastern Europe and Asia Pacific experienced an increase of 14 and 16 percentage points, respectively. For this investigation the analytical deductive method was used with the objective of contributing to the generation of protocols of action against fraud by presenting cases of fraud occurring in companies and a compilation of standards and publications that define the importance and role of the internal audit in the investigation of frauds.
\end{abstract}

\section{Keywords}

Transparency, fraud, impunity, protocol.

JEL Classification: H26, L2I, M42 


\section{Introducción}

De acuerdo a varias teorías y casos evidenciados, el fraude se encuentra en varias organizaciones que ingresan por diferentes formas; como manifiesta el Instituto de Auditores Internos de España (IAI, 2017, p.61): "Los fraudes son perpetrados por individuos y por organizaciones para obtener dinero, bienes o servicios, para evitar pagos o pérdidas de servicios, o para asegurarse ventajas personales o de negocio".

Estas acciones se consideran como un delito en los que una o varias personas atentan contra la propiedad o patrimonio de una institución; en la actualidad se han visto varios casos en los que los colaboradores de las diferentes instituciones han sido partícipes para realizar este tipo de delitos contra los bienes de las empresas. Esta grave situación se ha presentado por varias razones, entre ellas que no existe un adecuado control, establecer demasiado poder sobre un solo cargo, y la falta de capacitación al personal para poder reaccionar ante determinadas situaciones.

En los actuales momentos existe una creciente demanda social de mayor transparencia y control frente al fraude, así como una rendición de cuentas en los sectores tanto público como privado, lo que ha fomentado sanciones ejemplares en algunos

\section{Marco teórico}

De acuerdo a varios estudios realizados en diferentes países y organizaciones se ha determinado que el porcentaje de fraude ha ido creciendo durante los últimos años debido a varios aspectos internos y externos, los detallamos a continuación:

Según la Encuesta sobre fraude y delito económico 2018, de PriceWaterhouseCoopers México (2018, p. 16), del "porcentaje de organizaciones que experimentaron de- países. Esto también ha generado que las organizaciones y sus directores tengan la gran responsabilidad de establecer un proceso de investigación de cara a un eventual aparecimiento de esta índole.

Es por este motivo la importancia de llevar un control interno adecuado para las organizaciones, para evitar y prever cualquier tipo de delito contra las organizaciones, y es por eso que la auditoría interna cumple con un papel crucial, debido a que esta tiene el objetivo de llevar la evaluación del control que la empresa lleva, que permita cumplir con los objetivos y principios de la institución cumpliendo con las leyes, normas y políticas que la regulan, buscando la fiabilidad en la información de los saldos contables, estados financieros y la gestión que maneja la administración.

Esta investigación utiliza los métodos cualitativo exactamente el estudio de casos debido a que se toman una serie de situaciones y circunstancias que se centran en el método central del tema tratado, junto al método analítico porque aplicamos una evaluación de los casos e información obtenida para poder realizar un estudio de los fraudes y los pasos que se debe seguir para poder determinarlos, al igual que la forma en que la Auditoría Interna interviene.

litos económicos (2016-2018), el mayor incremento en delitos económicos se registró en América Latina, con un alza de 25 puntos porcentuales en comparación con el 2016". En México se registró una tendencia similar, con un aumento de 21 puntos porcentuales. "Países de Europa del Este y Asia Pacífico experimentaron un aumento de 14 y 16 puntos porcentuales, respectivamente". También se realizaron encuestas en las que se evidencia 
que los tipos de delitos económicos a nivel global categoriza entre las catorce tipologías, cuatro primeros lugares que son "la apropiación indebida de activos, soborno y corrupción, fraude cometido por el consumidor y delitos cibernéticos".

Estas cifras nos reflejan la importancia de llevar un adecuado control interno como se encuentra establecido en el Committee of Sponsoring Organizations of the Treadway Commission (COSO), que al detallar los cinco componentes de control interno, $y$ al hacer referencia al componente evaluación de riesgos, establece que: "El riesgo se define como la posibilidad de que un acontecimiento ocurra y afecte negativamente a la consecución de los objetivos". Entre los principios asociados al componente evaluación de riesgos tenemos el principio número 8, que establece: "La organización considera la probabilidad de fraude al evaluar los riesgos para la consecución de los objetivos" (COSO, 2013, p. 4).

Ernst \& Young (2016, p. 3), en su publicación Implementación efectiva de la nueva Guía Antifraude de COSO detalla: La "Guía de Gestión de Riesgos de Fraude (Guía Antifraude) se construye sobre la base del Marco Integrado de Control Interno COSO 2013" y es publicada por COSO en septiembre 2016 y La Asociación de Examinadores de Fraude Certificados (ACFE por sus siglas en inglés) es coautora de la Guía. Esta guía constituye un aporte fundamental para que las organizaciones evalúen en conjunto con Auditoría y equipos multidisciplinarios los riesgos de fraude.

El IAI (2015, p. 13), en su publicación Gestión del riesgo del fraude indica "Cualquier violación, desviación o infracción de un código de conducta o de cualquier control, debe ser reportado y tratado de forma oportuna, independientemente de quien lo cometa".

Es por ello que las empresas deben estar preparadas para actuar rápidamente frente a situaciones que comprometen los activos y su reputación, sin embargo, muchos profesionales actúan irresponsablemente cometiendo cualquier cantidad de errores que generalmente cuestan dinero y que inclusive podría ser mucho peor, ya que invalidarían las pruebas. Asimismo,

Es recomendable contar con protocolo de actuación frente al fraude. Entre otros aspectos el documento debe definir el procedimiento desde que se detecta la posible infracción hasta su resolución y tratar todos los aspectos que surjan a lo largo de la investigación: legales, toma de evidencias, consultas a externos, áreas involucradas, modelo de reporting, medidas correctivas, medidas disciplinarias. (IAI, 2015, p. 13)

Adicionalmente un protocolo de actuación frente al fraude define las responsabilidades de los involucrados en las tareas de investigación.

Los equipos de auditoría interna pueden desarrollar distintos roles en las tareas de investigación, desde la cooperación con equipos especializados internos o externos que lideran los trabajos, hasta asumir directamente la máxima responsabilidad en la investigación.

El IAI (2017, p. 7), en su publicación de las Normas Internacionales para el Ejercicio Profesional de la Auditoría Interna, y específicamente en las normas relacionadas sobre Atributos (1210.A2) establece:

Los auditores internos deben tener conocimientos suficientes para evaluar el riesgo de fraude y la forma en que se gestiona por parte de la organización, pero no es de esperar que tengan conocimientos similares a los de aquellas personas cuya responsabilidad principal es la detección e investigación del fraude.

Considerando la realidad actual, en donde los casos de fraude van en aumento, y los recursos disminuyen para la contratación de expertos, los nuevos roles de auditoría interna que demandan los directivos 
de las organizaciones es que los equipos de esta rama sean capaces de ofrecer respuestas adecuadas frente a casos de fraudes corporativos, o incluso asuman la máxima responsabilidad de la investigación.

El IAI (2017, p. 4), en su publicación de las Normas Internacionales para el Ejercicio Profesional de la Auditoría Interna, y específicamente en las normas relacionadas sobre Atributos (1000) establece: "La función de auditoría interna debe estar definida en propósito, autoridad y responsabilidades dentro del Estatuto"; por lo que en dicho Estatuto debe estar claramente definido el rol de

\section{Metodología}

Este trabajo de investigación es de carácter descriptivo, con un enfoque cualitativo (estudio de casos) y analítico; esto se debe a que mediante la evaluación de varias circunstancias (denominadas casos) se pueden determinar las causas y los efectos que se pueden llegar a determinar en cada caso dependiendo de los escenarios que se tome, en los que no se busca que los resultados sean generalizados. El objetivo del presente trabajo es contribuir a la generación de protocolos de actuación frente al fraude me- auditoría interna en relación al trato que se debe dar a los supuestos fraudes, delitos o irregularidades que se investiguen dentro de la organización, muy independiente de las responsabilidades que colateralmente tengan determinados departamentos de la organización, como por ejemplo, el departamento legal, recursos humanos o las propias áreas de negocio al momento de implementar medidas de control que mitiguen los riesgos identificados durante la investigación, y comunicados formalmente por el área de Auditoría Interna en su informe.

diante la presentación de casos específicos acontecidos en compañías. Adicionalmente se presenta una recopilación de estándares y publicaciones que define la importancia y el rol de la auditoría interna en la investigación de fraudes.

Hay que considerar también que en la actualidad las compañías solicitan una mayor participación del área de auditoría interna en el esclarecimiento de fraudes, apoyando a la generación de transparencia en las organizaciones.

\section{Resultados y discusión}

\section{¿Cómo realizar una investigación de fraudes?}

La implementación de un protocolo de actuación frente al fraude, constituye una buena práctica en las organizaciones, pues si bien es cierto presenta una serie de procedimientos a seguir desde que se detecta la posible infracción hasta que se toman las medidas correctivas y medidas disciplinarias, lo más importante es que se envía un mensaje a los empleados, accionistas, clientes y pro- veedores acerca de que en la organización no se toleran las acciones fraudulentas.

La investigación de indicios de fraude debe realizarse de una forma ordenada y sistemática, que permita recopilar las evidencias necesarias para identificar los autores materiales, cuantificación económica y modus operandi. Según Márquez (2018), la metodología para la auditoría forense puede dividirse en cinco fases: 
Figura 1. Fases de la auditoría

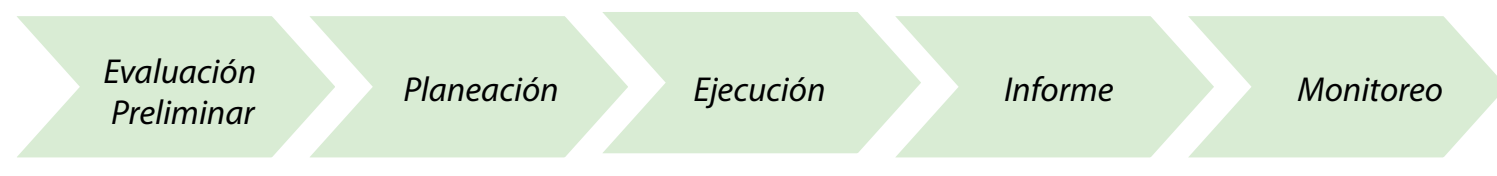

Fuente: Márquez (2018)

Adicionalmente, el IAI (2015, p. 122)

indica los aspectos a considerar por la alta

dirección en la investigación son:

Figura 2. Aspectos a considerar por la alta dirección en la investigación

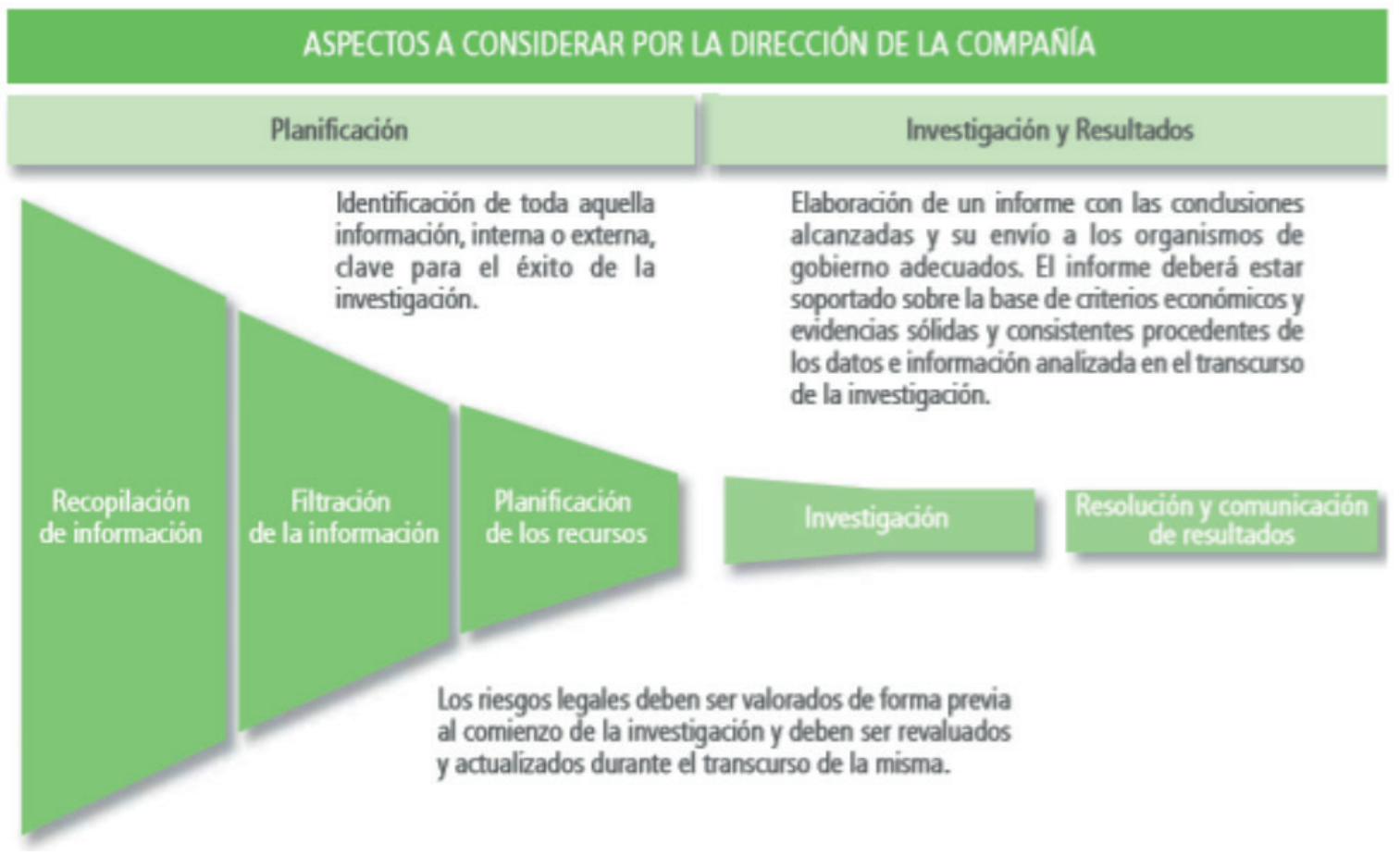

Fuente: IAI

El protocolo de actuación frente al fraude debe determinar las responsabilidades de los intervinientes, por lo que los equipos de auditoría pueden desarrollar varios roles en el proceso de investigación, desde el apoyo a equipos especializados en fraudes internos o externos hasta asumir la responsabilidad de la investigación.
Como lo establece el Manual de gestión de riesgos en el cuadro que antecede, los riesgos legales deben ser valorados de forma previa al comienzo de la investigación y deben ser revaluados y actualizados durante el transcurso de la misma, por lo que durante la planificación, ejecución y emisión de informes es importante tener la 
asesoría permanente de los abogados de la compañía.

Las organizaciones, a través de su gobierno corporativo, código de conducta y entes de cumplimiento deben transmitir un mensaje claro extensivo a los empleados, accionistas, clientes, proveedores que no existe tolerancia al fraude ni acciones ilícitas y por ende todas las denuncias de fraude deben tener un seguimiento a través de un protocolo de actuación frente al fraude; por lo que, como lo establece Ernst y Young (2016), en su publicación Implementación efectiva de la nueva Guía Antifraude de COSO en el principio 4:

La respuesta frente a reportes de fraude a través de "un sistema para la revisión, in- vestigación y resolución de situaciones de incumplimiento o denuncias de fraude y mala conducta de manera oportuna, competente y confidencial". Una organización debe tener protocolos de investigación y respuesta frente al fraude, iniciando con una cultura de "diga-algo-si-sabe-algo", y continuando con la clasificación de los reportes, gestión de casos, aseguramiento de la evidencia, asesoría legal y de contabilidad forense, análisis de causa raíz y remediación de controles. (Ernst \&Young, 2016, p.7)

Para esta investigación se tomó en consideración diferentes tipos de categorías de fraudes; esto es importante para determinar en qué situación se puede encontrar la organización y así saber cómo poder actuar.

Figura 3. Categorías de fraude

\begin{tabular}{|c|c|}
\hline CATEGORÍA & DETALLE \\
\hline $\begin{array}{l}\text { Reporte fraudulento de información } \\
\text { financiera }\end{array}$ & $\begin{array}{l}\text { Cualquier declaración errónea intencional de información contable } \\
\text { constituye un reporte fraudulento de información financiera. }\end{array}$ \\
\hline $\begin{array}{l}\text { Reporte fraudulento de información no } \\
\text { financiera }\end{array}$ & $\begin{array}{l}\text { Reporte fraudulento de riesgos y esquemas no financieros que podrían } \\
\text { dar lugar a falsedad en los reportes de información ambiental, seguri- } \\
\text { dad, control de calidad o métricas operativas. }\end{array}$ \\
\hline Malversación de activos & $\begin{array}{l}\text { Por parte de empleados, clientes o proveedores, u organizaciones crimi- } \\
\text { nales afectando los activos tangibles e intangibles de la organización, } \\
\text { así como las oportunidades de negocio. } \\
\text { Algunos ejemplos incluyen: } \\
\text { - Robo de empleados. } \\
\text { - Facturas de proveedores ficticios. } \\
\text { - Falsas reclamaciones de clientes. } \\
\text { - Ciberataques externos. }\end{array}$ \\
\hline Otros actos ilegales y de corrupción & $\begin{array}{l}\text { Constituyen actos ilegales: "violaciones a las leyes o regulaciones gu- } \\
\text { bernamentales que podrían ocasionar un impacto material, directo o } \\
\text { indirecto, en los reportes financieros externos". } \\
\text { Como ejemplos se incluyen el soborno, instigación, encubrimiento o } \\
\text { complicidad en el fraude, violación a las leyes, uso ilícito de información } \\
\text { personal, secretos comerciales o información de seguridad nacional; } \\
\text { violaciones de carácter laboral, de exportación de tecnología o leyes de } \\
\text { protección al consumidor. } \\
\text { La corrupción se define como "el mal uso del poder delegado para la } \\
\text { obtención de un beneficio privado" (p.ej. violaciones del U.S. Foreign Co- } \\
\text { rrupt Practices Act (FCPA por sus siglas en inglés) o leyes similares de } \\
\text { otros países). }\end{array}$ \\
\hline
\end{tabular}

Fuente: Ernst \& Young (2016) 


\section{Estudio de casos}

Al ejecutar la investigación de fraude para dos casos puntuales acontecidos en una compañía podremos identificar los autores materiales, la cuantificación económica

\section{Caso 1}

\section{Modus operandi}

El sistema contable tenía una transacción para la emisión de un documento utilizado para registrar los comprobantes de retención y para generar notas de crédito por descuentos; las transacciones de notas de crédito fueron delegadas por el Jefe de Agencia al Cajero para que le apoye con la generación de notas de crédito, con el propósito de hacer más eficiente el proceso. Esta decisión no fue comunicada a las áreas de control.

El cajero utilizaba esta transacción para dar de baja la cartera y tomar el dinero correspondiente de la cobranza, estas notas de crédito afectaban directamente a una cuenta de gastos de ventas que pasó desapercibida por un largo periodo pues los valores cargados eran pequeños, sin embargo, el perpetrador decidió cada vez aumentar los valores pues se dio cuenta de que no existía ninguna observación de las áreas involucradas.

Adicionalmente, esta transacción no aparecía en el cierre de caja, pues no le era

\section{Caso 2}

\section{Modus operandi}

La instalación de ubicaciones logísticas en una compañía fue realizada por empleados de una compañía, sin embargo, la factura pertenecía a otra compañía, lo cual llamó la atención del gerente de logística encargado y notificó este particular a los entes de control.

El área de Auditoría visitó el domicilio del proveedor que emite la factura que era (dato referencial para efectos de este artículo), el modus operandi y las deficiencias de control interno identificadas que sirvieron de base para fortalecer los controles:

propia, ya que el cajero no era responsable de generar este tipo de notas de crédito.

El Gerente encargado de la cuenta de gastos, mediante la revisión de los valores presupuestados versus los ejecutados de la cuenta del gasto, observó valores importantes que afectaban a su cuenta de gastos sin ninguna justificación, por ello solicitó respaldos.

La investigación en este caso fue liderada por el área de Auditoría Interna para lo cual se planificó el protocolo a seguir y se solicitó el soporte de los abogados de la compañía desde el inicio, ejecución de la investigación y cierre de la misma con la entrega del informe, evidencias a los abogados para que gestionen el juicio correspondiente.

La falla de control más importante en este caso es la inadecuada segregación de funciones y la falta de revisión de los roles y perfiles en el sistema financiero por un ente independiente.

una casa, no una oficina, ni talleres; la compañía, de acuerdo a sus ingresos, no tenía declaraciones de impuestos importantes. Se verificó en la Superintendencia de Compañías y la empresa tenía como socio al responsable de compras.

Las ubicaciones logísticas tenían el logo de una compañía conocida en el mercado, por lo que el equipo de auditoría visitó 
a dicha empresa y solicitó la oferta presentada a la compañía que facturó. El valor era inferior al facturado y las especificaciones técnicas eran inferiores.

La investigación de este caso fue liderada por el área de Auditoría Interna para lo cual se planificó el protocolo a seguir y se solicitó el soporte de los abogados de la compañía desde el inicio, ejecución de la investigación y cierre de la misma

\section{Caso 3}

Para poder continuar con el estudio, también se tomó en consideración el caso que la firma Iberaudit Kreston manifiesta en su publi-

\section{Modus operandi}

Uno de los principales costos de la empresa son las refacciones y mantenimiento de las ambulancias. Para gestionarlos la empresa cuenta con un software donde a cada ambulancia se le identifica por un número al cual se le cargan todas las refacciones y mantenimientos realizados a las mismas. Todo repuesto utilizado debía tener asignada una unidad o caso contrario debía mediar la autorización de un gerente de taller.

El personal de auditoría hacía recuentos físicos periódicos con el objeto de verificar existencias de faltantes de stock, los mismos siempre eran satisfactorios ya que el inventario físico siempre coincidía con el teórico arrojado por el sistema.

Ahora bien, un grupo de mecánicos advirtieron que si bien el sistema funcionaba correctamente no había controles que consideraran la razonabilidad ni de detalle sobre las refacciones y mantenimientos realizados a las distintas unidades. De esta forma comenzaron a realizar 'arreglos ficticios' con el objeto de sustraer repuestos y venderlos en el mercado paralelo. Es así que se identificaron casos que a una misma unidad se le cambiaron dos y hasta tres veces al año las cubiertas o la batería. Esta situación se daba en ma- con la entrega del informe, evidencias a los abogados para que gestionen el juicio correspondiente.

La falla de control más importante en este caso fue un exceso de confianza al entregar al gerente el poder para liderar estos proyectos de inversión el proceso de compras, lo cual también demuestra inadecuada segregación de funciones, así como una falta de calificación de proveedores.

cación La auditoría interna y la prevención del fraude.

yor medida en algunas unidades donde los mecánicos habían llegado a un 'arreglo' con los choferes para que ellos presenten las solicitudes de reparación o cambios de piezas y de esta forma viabilizar la irregularidad. Esta situación irregular se advirtió por una denuncia anónima utilizando el sistema de denuncias implementado por la compañía.

Este desfalco ocurrió por las siguientes situaciones que se dieron en forma concomitante:

Ausencia de adecuados procedimientos de control para la aprobación del uso de los repuestos. Ausencia de supervisión por parte del jefe de repuestos.

Ausencia de adecuados controles por oposición de intereses. Nadie de otros sectores realizaba controles de razonabilidad sobre los consumos de repuestos de las ambulancias.

Ausencia de ambiente de control. La falta de un ambiente de control facilitó la comisión de las irregularidades ya que los mecánicos al verificar que nadie estaba controlando los consumos de repuestos vieron facilitado su accionar delictivo. (Iberaudit Kreston, 2013, p. 34-35) 


\section{Análisis general de casos}

El riesgo que corre una institución de sufrir un fraude por diversas razones y, especialmente, por el entorno en que se encuentra es muy alta, ya que sus actividades diarias dan lugar a un porcentaje de riesgo; si la empresa no posee un correcto control interno en las diferentes áreas y de acuerdo a las funciones establecidas, no se podrá determinar el fraude al que enfrentará o se encuentra atravesando, porque como podemos ver los resultados negativos o consecuencias se determinarán de forma tardía. Es por esta razón que la audi- toría interna cumple un papel de suma importancia en las organizaciones, ya que su función es supervisar y controlar los riesgos a los que va a enfrentar la empresa, determinando así la forma de prevenir pérdidas o problemas legales que se puedan presentar.

Una correcta auditoría interna llevará un adecuado control interno y sus componentes de acuerdo a la aplicación que tenga la empresa, con ello disminuyen los porcentajes de riesgos que enfrenta toda institución, incluido el fraude.

\section{¿Cuál es el rol de la auditoría interna en la investigación de fraudes?}

\section{Perfil de un auditor interno}

Los auditores internos deben tener conocimientos suficientes para evaluar el riesgo de fraude y la forma en que se gestiona por parte de la organización, pero no es de esperar que tengan conocimientos similares a los de aquellas personas cuya responsabilidad principal es la detección e investigación del fraude. (IAI, 2017, p. 7)

Y también podemos considerar lo manifestado por el Dr. Lugo - publicado en la página United States InterAmerican Community Affairs (2005) - que en su libro Auditoría forense, una perspectiva de investigación científica, establece que:

El auditor es un investigador, es un analista, un técnico o profesional especializado en un área determinada y por su carácter de experto puede establecer las causas, los efectos las consecuencias, incluso los móviles que ha tenido un hecho específico; es obligación de un auditor generar un informe de su gestión y si en su investigación descubre un delito o sospecha de que se está cometiendo un hecho doloso, debe informarlo por escrito a quien lo haya contratado y hacer un seguimiento para que se tomen las medidas legales corres- pondientes y si ese 'hecho' o delito es en detrimento de 'otros' como clientes o entidades debe reportarlo a las autoridades so pena de convertirse en un encubridor o cómplice de un delito criminal por omisión o negligencia. (Lugo, 2005, parr.1)

Considerando el avance tecnológico en el quehacer de los negocios y la tendencia actual a la implementación de tecnologías disruptivas, es muy probable que en las investigaciones de fraude sea necesario la aplicación de procedimientos específicos para la recopilación de evidencia digital, por lo que, si el equipo de auditoría no dispone de los medios o de las competencias técnicas necesarias, se deberá contratar expertos en el área de TIC, por lo que de acuerdo con el Instituto de Auditores Internos (2017, Norma 1210.A1): "El Director de Auditoría Interna debe obtener asesoramiento y asistencia competente en caso de que los auditores internos carezcan de los conocimientos, las aptitudes u otras competencias necesarias para llevar a cabo el trabajo".

El Dr. Lugo también establece un concepto desde la perspectiva científica de 
quién debe ser un auditor, investigador, forense y la forma en que debe preparase.

[...] El auditor-investigador-forense, debe ser un profesional integral, abierto a la innovación, al trabajo de grupo con profesionales o técnicos de diferentes disciplinas, sin importar cual se su especialidad, debe prepararse también en el campo de la psicología, entiéndase bien, no obtener un título como psicólogo, pero al menos tener una base de la psicología del ser humano, los diferentes comportamientos, las conductas, las tendencias, los estilos de vida, los grupos y clases sociales y quizás lo más importante, aprender a distinguir los perfiles de los posibles sospechosos de cometer actos ilícitos y su modus operandi. (Lugo, 2005, parr.16)
Adicionalmente, el acompañamiento del profesional legal en sus diferentes especializaciones de derecho como laboral, penal, entre otros, es fundamental para evitar cometer errores y que dichas pruebas no sean aceptadas para efectos legales. Por esto, el IAI (2015, p. 78) establece: "[...] los marcos legales se hacen más estrictos y han incluido en últimas fechas la tipificación de fraudes fiscales, los abusos de confianza, el lavado de dinero y han puesto especial énfasis en el delito de 'guante blanco'".

La Norma Internacional de Auditoría (NIA) 240, en los párrafos 4 y 5 establece:

Responsabilidad en relación con la prevención y detección del fraude

1. Los responsables del gobierno de la entidad y la dirección son los principales responsables de la prevención y detección del fraude. Es importante que la dirección, supervisada por los responsables del gobierno de la entidad, pongan gran énfasis en la prevención del fraude, lo que puede reducir las oportunidades de que este se produzca, así como en la disuasión de dicho fraude, lo que puede persuadir a las personas de no cometer fraude debido a la probabilidad de que se detecte y se sancione. Esto implica el compromiso de crear una cultura de honestidad y comporta-

\section{Responsabilidad del auditor}

2. El auditor que realiza una auditoría de conformidad con las NIA (Normas Internacionales de Auditoría) es responsable de la obtención de una seguridad razonable de que los estados financieros considerados en su conjunto están libres de incorrecciones materiales debidas a fraude o error. miento ético, que puede reforzarse mediante una supervisión activa por parte de los responsables del gobierno de la entidad. La supervisión por los responsables del gobierno de la entidad incluye prever la posibilidad de elusión de los controles o de que existan otro tipo de influencias inadecuadas sobre el proceso de información financiera, tales como intentos de la dirección de manipular los resultados con el fin de influir en la percepción que de ellos y de la rentabilidad de la empresa tengan los analistas. (International Federation of Accountants, 2013, párr. 4)

Debido a las limitaciones inherentes a una auditoría, existe un riesgo inevitable de que puedan no detectarse algunas incorrecciones materiales en los estados financieros, incluso aunque la auditoría se haya planificado y ejecutado adecuadamente de conformidad con la Norma Internacional de Auditoría (NIA) 
240, "Responsabilidades del auditor en

la auditoría de estados financieros con respecto al fraude". (International Federation of Accountants, 2013, p. 3)

\section{Rol del auditor interno}

Después del análisis de los diferentes conceptos expuestos del perfil y responsabilidades que debe tener el auditor interno, se debe analizar la importancia que tiene su rol en el momento de detección y prevención a los fraudes, debido a que un profesional bien capacitado, con las correctas actitudes y aptitudes podrá llegar a determinar el riesgo que atraviesa la empresa ante la posible situación de un fraude por las diferentes circunstancias que puede presentar.

El rol del auditor interno es de suma importancia para determinar y reducir al mínimo el riesgo que corre la institución, si no existe una parte encargada de controlar y evaluar la situación que atraviesa cada de-

\section{Conclusiones}

- Las organizaciones han implementado evaluaciones de riesgo, adoptado programas de ética y cumplimiento, política anti fraude, elaborado códigos de conducta, sin embargo, el incremento de delitos, según la encuesta sobre fraude y delito económico 2018, edición México de PriceWaterhouseCoopers, indica que el mayor incremento en delitos económicos se registró en América Latina, con un alza de 25 puntos porcentuales en comparación con 2016.

- Un tema muy grave para las organizaciones y tan fuerte como el fraude mismo es la impunidad, por lo que el castigo a los actos irregulares no debería ser la excepción, sino la regla, obviamente siguiendo el debido proceso y considerando todas las normas legales aplicables.

- Según la Encuesta sobre fraude y delito económico 2018, edición México partamento o área de la empresa, la oportunidad para que se desarrolle una situación de fraude es sumamente alta, y sin posibilidad de ser detectada, hasta llegar a consecuencias graves para la organización.

El profesional debe realizar pruebas correspondientes a cada área, sus funcionarios y recursos para determinar las correctas actuaciones, para así reportar cualquier irregularidad y prevenir consecuencias negativas, deberá determinar que toda actividad, contrato y utilización de recursos se encuentra bajo la normativa legal del país y de la institución, lo que permite evitar que se llegue al fraude, presentando los hallazgos después de haber realizado las pruebas correspondientes.

de PriceWaterhouseCoopers, se detalla que: Un programa de cumplimiento debe tener un balance entre componentes de disuasión, prevención, detección, investigación y sancionamiento, así como fortalecer los diversos aspectos que impliquen gente, procesos y tecnología

- La investigación de fraudes debe estar soportada por protocolos de actuación frente al fraude, en la que se determine las responsabilidades de los involucrados. Es indispensable que los equipos de auditoría puedan desarrollar varios roles en el proceso de investigación; desde la cooperación con equipos especializados internos o externos que lideran los trabajos, hasta asumir directamente la máxima responsabilidad. Dicho proceso de investigación debe estar acompañado por la asesoría de los abogados de las organizaciones. 
- En la era que vivimos, los perpetradores pueden apoyarse en herramientas tecnológicas para cometer fraudes, por lo que es necesario que profesionales informáticos formen parte de los equipos especializados de investigación.

- Las organizaciones cada vez esperan que los auditores internos jueguen un papel protagónico en la detección e investigación de fraude, a pesar de que las Normas Internacionales para el Ejercicio Profesional de la Auditoría Interna establecen que los auditores internos deben tener conocimientos suficientes para evaluar el riesgo de fraude y la forma en que se gestiona por parte de la organización, pero no es de esperar que tengan conocimientos similares a los de aquellas personas cuya responsabilidad principal es la detección e investigación del fraude.

\section{Referencias}

Association of Certified Fraud Examiners (ACFE) (2018). Reporte a las naciones sobre el abuso y el fraude ocupacional. Obtenido de http://www.acfe.com/report-to-thenations/2018/

Committee of Sponsoring Organizations of the Treadway Commission (COSO) (2013). Control Interno-Marco Integrado. Obtenido de http://doc.contraloria.gob.pe/ControlInterno/Normativa_Asociada/coso_2013resumen-ejecutivo.pdf

Committee of Sponsoring Organizations of the Treadway Commission (2016). Risk Management Guide. Obtenido de https://www.coso. org/Documents/COSO-Fraud-Risk-Management-Guide-Executive-Summary.pdf

Ernst \& Young (2016). Implementación efectiva de la nueva Guía Antifraude de COSO. Obtenido de https://docplayer.es/74825089Implementacion-efectiva-de-la-nuevaguia-antifraude-de-coso.html

Iberaudit Kreston. (2013). La auditoría interna y la prevención del fraude. Obtenido de http://www.kreston.es/public/uploads/ wysiwyg/files/Ebook\%20IBERAUDIT\%20 Kreston.pdf

International Federation of Accountants (IFAC) (2013). Normas Internacionales de Auditoría (NIA). Obtenido de https://www.ifac. org/publications-resources/normas-internacionales-de-auditor-isa-presentaciones-isa-m-dulo-de-powerpoint
Institute of Internal Auditors (2017). The Institute of Interna Auditors. Obtenido de https:// na.theiia.org/Pages/IIAHome.aspx

Instituto de Auditores Internos de España (IAI) (2015).Gestióndelriesgodelfraude:Prevención, detección e investigación. Obtenido de https://auditoresinternos.es/uploads/ media_items/f\%C3\%A1brica-fraude.original.pdf

Instituto de Auditores Internos de España (2017). Normas Internacionales para el Ejercicio profesional de la Auditoría Interna. Obtenido de https://auditoresinternos.es/sobrenosotros/la-profesi\%C3\%B3n/normas

Instituto de Auditores Internos de España (2015). Manual de gestión de riesgos. Obtenido de HYPERLINK "https://bookstore.theiia. org/manual-de-gesti\%C3\%B3n-delriesgo-de-fraude-prevenci\%C3\%B3n-2" https://bookstore.theiia.org/manual-degesti\%C3\%B3n-del-riesgo-de-fraudeprevenci\%C3\%B3n-2

Lugo, D. (2005). United States InterAmerican Community Affairs. Instituto de Auditores Forenses. Obtenido de http://www.ideaf.org

Márquez, R. (2018). Auditoría forense. Ciudad de México: Azucena García Nares. PriceWaterhouseCoopers (2019). Encuesta sobre fraude y delito económico. Obtenido de https://www.pwc.com/mx/es/publicaciones/c2g/2018-04-13-encuesta-delitoseconomicos-2018-mexicov4.pdf 